\title{
L'évolution des comportements d'achat à Prague : du communisme au fun shopping
}

Evolution of shopping habits in Prague from communism to fun shopping

\section{Carole Pommois}

\section{(2) OpenEdition}

12 Journals

\section{Édition électronique}

URL : http://journals.openedition.org/belgeo/10864

DOI : 10.4000/belgeo.10864

ISSN : 2294-9135

Éditeur :

National Committee of Geography of Belgium, Société Royale Belge de Géographie

Édition imprimée

Date de publication : 30 juin 2006

Pagination : 9-26

ISSN : 1377-2368

\section{Référence électronique}

Carole Pommois, «L'évolution des comportements d'achat à Prague : du communisme au fun

shopping », Belgeo [En ligne], 1-2 | 2006, mis en ligne le 18 décembre 2013, consulté le 01 mai 2019. URL : http://journals.openedition.org/belgeo/10864 ; DOI : 10.4000/belgeo.10864

Ce document a été généré automatiquement le 1 mai 2019.

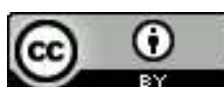

Belgeo est mis à disposition selon les termes de la licence Creative Commons Attribution 4.0 International. 


\section{L'évolution des comportements d'achat à Prague : du communisme au fun shopping}

Evolution of shopping habits in Prague from communism to fun shopping

\section{Carole Pommois}

La recherche présentée dans cet article a été financée par une bourse Marie Curie de la Commission Européenne (2003-2004).

\section{Introduction}

1 En 1997, vers la fin de l'année, le premier centre commercial, ancré par un hypermarché, a ouvert ses portes dans l'une des cités-dortoirs de la période communiste, à la périphérie de Prague. Cette ouverture allait inaugurer une ère nouvelle pour le commerce de détail pragois et pour la consommation. Depuis, les centres et les parcs commerciaux ont fleuri tout autour de la capitale. De nouveaux concepts, développés dans les pays occidentaux et importés en Europe centrale (comme en Europe du Sud) ont fait une entrée remarquée. Les consommateurs, avides de ces nouvelles expériences commerciales, symbolisant le mode de vie occidental, ont vite répondu à l'appel, modifiant les pratiques urbaines et sociales. La question à l'ordre du jour est la même que celle que les sociologues d'Europe de l'Ouest se posent: les centres commerciaux vont-ils ou sont-ils déjà entrain de remplacer le centre-ville traditionnel comme lieu de socialisation? Les centres commerciaux sont-ils devenus des lieux de promenade comme le rappelle la terminologie anglo-saxonne shopping mall où mall signifie promenade? Comment les comportements d'achat des habitants de Prague évoluent-ils? Est-il pertinent d'exprimer cette problématique en termes de répulsion-attraction vis-à-vis de ces centres commerciaux qui mettent en péril la survivance du petit commerce indépendant? Alors que le shopping moderne s'est installé très lentement en Europe occidentale depuis les années 1960-70, il met moins de 10 ans à s'implanter en Europe centrale (et en Europe du Sud dont les villes 
comme Barcelone ou Lisbonne se dotent d'équipements commerciaux modernes, répondant au concept de fun shopping).

Une première partie est consacrée à l'évolution de l'organisation spatiale du commerce de détail pragois, depuis le début des années 1990 à nos jours, où en seront énumérées les principales phases. Une seconde partie s'intéressera plus précisément aux comportements d'achat des habitants de Prague, s'appuyant sur une enquête réalisée auprès d'un échantillon de 400 habitants. Cette enquête permet de saisir l'évolution du comportement spatial des consommateurs, en rapport avec la modernisation et l'internationalisation de la petite distribution, dans un environnement marqué par l'absence de législation restrictive sur l'ouverture des grandes surfaces.

\section{La petite distribution : concentration et internationalisation}

3 Novembre 1989, la Révolution de Velours met fin à la période communiste en Tchécoslovaquie. Une nouvelle époque s'ouvre avec ses promesses et ses difficultés. Les principes du marché sont rétablis, l'économie s'ouvre aux marchés occidentaux, le rôle de l'Etat dans la marche de l'économie est destiné à se minimiser peu à peu. Pour ce faire, des processus de restitution, de privatisation et de dérégulation sont engagés. En Tchécoslovaquie, la préférence est pour le gradualisme, mais dans la réalité, ardu à appliquer et remplacé par des thérapies de choc. C'est en ces termes que les économistes décrivent les moyens choisis par les Etats pour engager les anciens pays communistes européens dans la voie du libéralisme. Les biens autrefois confisqués et les entreprises socialisées sont rendus au secteur privé. De ce secteur émerge un marché immobilier et foncier renaissant. De ces phénomènes découleront des processus de mutation des espaces, notamment de celui qui nous intéresse, l'espace intra urbain, touchant toutes les fonctions urbaines à des vitesses différentes. C'est ce que nous appellerons la période de transition bien que le terme même de transition soit remis en cause par les économistes aujourd'hui. L'on parle aussi de plusieurs transitions qui ont lieu à des vitesses différentes selon les secteurs économiques, politiques et sociaux. Dans le cas des espaces urbains, les premiers effets visibles dans le paysage concernent le commerce de détail.

\section{La petite distribution sous le régime communiste}

Contrairement aux pays communistes voisins, en Tchécoslovaquie, le secteur de la distribution fut entièrement nationalisé. Entièrement contrôlé et dirigé par l'Etat, il était organisé en grands secteurs, chacun correspondant à un type de bien (les fruits et légumes, les vêtements, les chaussures...). Ces grands secteurs constituaient des grandes entreprises d'Etat, placées directement sous l'autorité du Ministère du commerce intérieur et du tourisme. Elles étaient organisées hiérarchiquement. Les commerçants qui tenaient les commerces, étaient devenus des employés de l'Etat, et n'avaient donc plus de soucis de rendement. Les petits commerces étaient destinés en général à écouler les biens produits dans l'industrie publique. La localisation des établissements commerciaux était décidée par l'administration selon un modèle qui peut rappeler la théorie des places centrales. Les commerces étaient répartis de manière à desservir la population à diverses échelles administratives, du micro quartier aux grands centres urbains. Les prix pratiqués étaient les mêmes partout dans le pays. Vers la fin des années 1980, à l'époque où M. Gorbatchev encouragea l'introduction de mécanismes de marché mais sans remettre en 
cause l'économie centralement planifiée, ces mêmes mécanismes furent introduits en Tchécoslovaquie, en vue de redynamiser une économie en difficulté. La petite distribution fut l'objet d'une déconcentration et une forme de propriété privée fut admise, bien que limitée à l'artisanat. Il s'agissait aussi de réagir contre le marché noir qui avait pris des dimensions internationales. Et c'était là, l'un des paradoxes de l'économie centralement planifiée : les rayons sont vides, mais les frigos sont pleins. Si les longues queues à l'entrée des magasins et les rayons vides, sont devenus le symbole de la petite distribution sous le régime communiste, ce n'est pas forcément parce qu'il y avait pénurie. La cause en était généralement une mauvaise gestion des circuits de distribution.

5 Au début de la transition, il fut décidé par les autorités tchèques, de procéder à la privatisation à l'échelle de l'établissement commercial, soit à la base de la structure pyramidale des grandes entreprises publiques. Les petits commerces furent soumis au processus de la petite privatisation par l'organisation de ventes aux enchères. Il y eut deux rounds, au cours desquels tout citoyen tchécoslovaque majeur pouvait participer. La participation étrangère a été interdite lors du premier tour, afin d'éviter que toute l'économie tchécoslovaque ne passe aux mains étrangères. Une partie du petit commerce de détail put retourner directement aux mains des anciens propriétaires par le biais de la restitution. Les grands magasins et les hôtels furent par contre soumis au processus de la grande privatisation.

\section{Dès le début des années 1990 : l'arrivée des premières marques occidentales}

6 Tout au début de la période de transition, il existait donc deux systèmes de propriété qui cohabitaient. Les fermetures furent assez nombreuses. Dans le centre-ville, les quartiers éloignés des principales artères connurent un phénomène de dépréciation dont il subsiste encore quelques rares traces au début des années 2000. La restitution et la privatisation s'étant réalisées au pied de la pyramide, la petite distribution du début des années 1990 se caractérise par sa très grande fragmentation. Le petit commerce indépendant est la règle. Cependant, le réseau de supermarché qui existait déjà, est privatisé et repris par des distributeurs allemands, autrichiens ou belges (Julius Meinl, Delvita...) Si concentration il $\mathrm{y}$ a, elle s'opère dans un premier temps dans le secteur alimentaire et ne concerne pas encore les petits commerces. Le petit commerce se caractérise aussi par sa courte durée de vie. Les Tchèques n'ont guère l'expérience des affaires, et ils doivent vite apprendre les lois de la concurrence. Afin d'y survivre, les nouveaux commerçants inventent toutes sortes de formules parfois insolites, mais s'étonnent encore quand, dans la même rue, un autre commerçant vient proposer la même activité. Au début, le petit commerce n'est pas spécialisé. Cette spécialisation ira en s'accroissant par la suite. Les premières enseignes occidentales arrivent à Prague à partir de 1992. Sous le régime communiste, les produits n'avaient pas de marque, et les commerces n'avaient pas d'appellation spécifique permettant de les distinguer. Ils portaient tout simplement le nom du type de produits vendus (par exemple Ovoce-Zelenina pour les fruits et légumes, Odevy pour les vêtements). Les premières marques que les Tchèques reconnaissent sont occidentales. Jusqu'en 1989, les produits étrangers étaient accessibles, mais uniquement dans des établissements spéciaux de la chaîne Tuzex et contre des devises étrangères. 


\section{Les commerçants versus les vendeurs des rues}

7 Le nombre des établissements commerciaux s'accroît très rapidement. Parallèlement, d'autres formes de commerces qui existaient déjà pendant la période communiste (et qui sont comptabilisées par les recensements annuels jusqu'en 1989) continuent de se développer. Il s'agit des kiosques, des marchés et des vendeurs des rues. Prague a vu aussi l'arrivée de vendeurs des rues de nationalité russe, et la presse du début des années 1990 ne manque pas d'exemples d'altercations entre les vendeurs tchèques et les vendeurs russes. Dans le même temps, ces vendeurs de plus en plus nombreux dans les rues du centre-ville de Prague sont vus comme une concurrence déloyale par les principales enseignes présentes. En 1993, une association de distributeurs, s'est constituée pour porter une action contre cette forme de vente et réclamer la régulation de cette activité, en particulier sur le pont Charles. Les vendeurs des rues qui exerçaient leur activité sur le pont y ont vu là une atteinte à leur liberté. Malgré cela, la ville de Prague a pris la décision de réguler les activités économiques sur le pont Charles et d'en confier même la sécurité à une milice privée. De jour, seuls les vendeurs, ayant une licence, peuvent s'y installer. De nuit, les Tchèques, surtout des jeunes, reprennent possession du pont ${ }^{1}$.

\section{Un renforcement de la centralité du centre-ville}

8 Si le petit commerce est au début très peu spécialisé, une spécialisation s'opère néanmoins dans le centre-ville. Prague est une ville à destination touristique. Le tourisme international a été encouragé et développé dès les années 1980, encore sous le régime communiste, où une restauration de façade fut opérée afin d'améliorer le visage de la ville. Dans les années 1990, les rues du centre de la ville se spécialisent de plus en plus vers cette activité (Figure 1). Les Pragois qui résident à Prague et dans son centre-ville craignent cette nouvelle évolution et considèrent avec appréhension la disparition du petit commerce de proximité. Les résidents ont peur que la ville leur échappe. Les autorités de la ville partagent cette crainte et le Bureau du Chef Architecte de Prague ${ }^{2}$ analyse toutes les formules possibles afin d'éviter que le centre historique de Prague ne se transforme en musée et ne se vide de ses habitants. Pour le moment, les résidents du centre-ville sont essentiellement des personnes âgées. Ils sont soumis à plusieurs phénomènes qui tendraient à les chasser du centre-ville: d'une part la restitution des immeubles à leurs anciens propriétaires ou descendants et d'autre part la libéralisation du marché immobilier ${ }^{3}$. Cette question est également vue comme un problème aux yeux des planificateurs car les loyers protégés ne fournissent pas les revenus nécessaires aux investissements qu'il faudrait engager pour la restauration du bâti. Cependant, s'agissant de conserver au centre-ville une certaine fonction résidentielle, une autre mesure a été adoptée, celle-ci concernant les activités commerciales. Le nouveau propriétaire immobilier s'engage à louer l'espace commercial au commerçant qui y exerce son activité, pour au moins une durée de 5 ans. C'était une mesure destinée à maintenir dans le centreville un commerce de proximité. Mais la spécialisation grandissante du centre historique vers le tourisme international poursuit son chemin. 
Figure 1. Le commerce de détail dans les quartiers centraux de Prague.

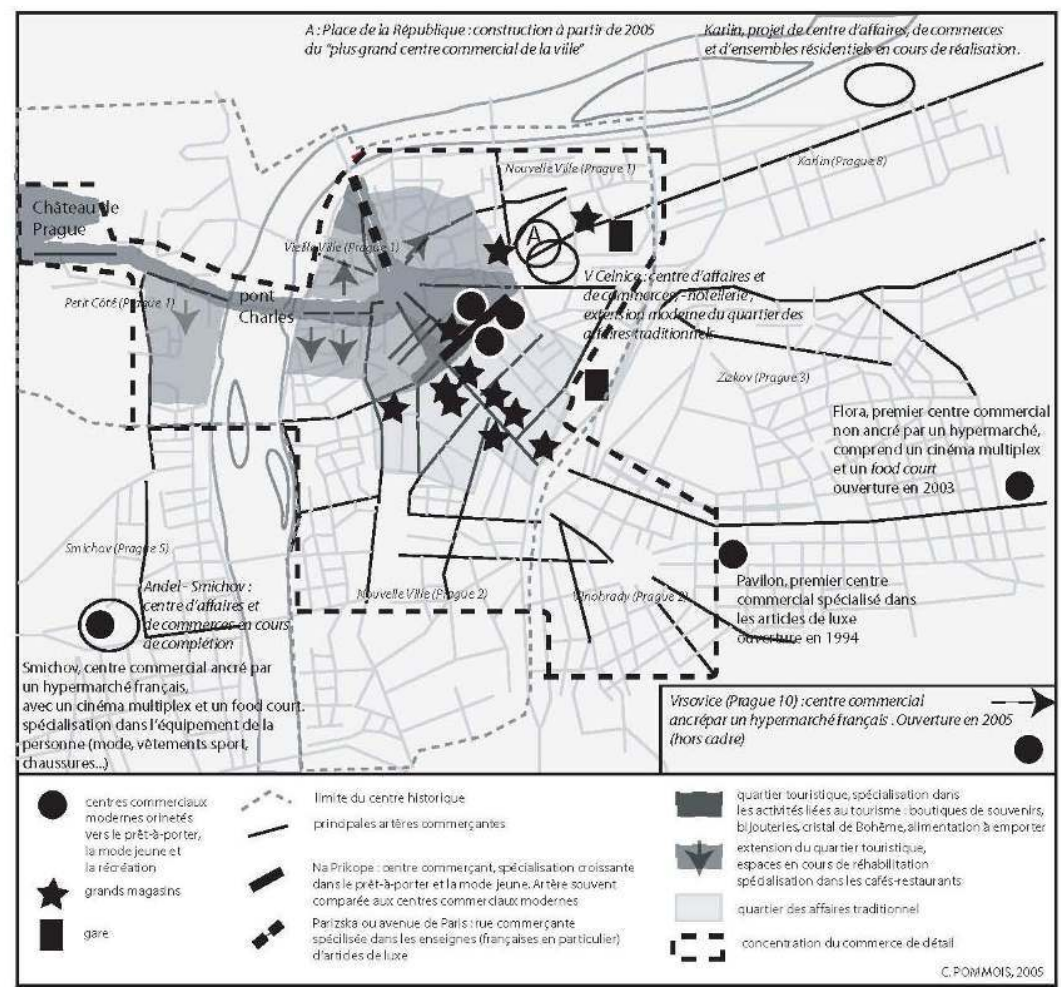

Le nombre des établissements commerciaux s'accroît rapidement partout mais, si l'on analyse leur distribution, on ne peut que constater le poids grandissant du centre historique, qui se confond avec le centre traditionnel des affaires. Le centre-ville de Prague ne souffre d'aucune concurrence encore jusqu'au milieu des années 1990. L'organisation spatiale du commerce de détail pragois se caractérise par sa structure hiérarchique et la forte prédominance du centre-ville. Celui-ci est le seul à accueillir les distributeurs occidentaux, et il concentre les emplois de bureaux avec l'arrivée des firmes occidentales et aussi avec le développement des activités de services destinés aux entreprises. Cette double concentration, commerces et bureaux, accentue le phénomène des migrations pendulaires de la périphérie vers le centre-ville et aboutit à une saturation des transports en commun ainsi qu'à l'apparition des embouteillages, consécutifs au développement du parc automobile.

\section{Les centres commerciaux dans les quartiers centraux}

En 1994, une nouvelle phase de l'évolution du commerce de détail pragois s'amorce avec l'ouverture, dans l'un des faubourgs de la fin du XIX siècle, du premier centre commercial concentrant des boutiques de luxe. Dans le centre-ville, les opérations de réhabilitations se poursuivent. Les passages retrouvent leur fonction commerciale (Figure 2) et les petits commerces ne cessent de fermer et d'ouvrir. C'est en 1996 qu'un second centre commercial, associé à la fonction des affaires, ouvre ses portes dans la principale artère du centre-ville Na Prikope (rue des douves), devenue le véritable coeur commercial de Prague. Depuis, les projets de réhabilitation se bousculent et l'on voit la multiplication des centres commerciaux et des grands magasins. Jusqu'à cette période, le centre-ville de Prague demeure le lieu d'implantation privilégié des grandes marques internationales. 
Cette préférence pour le coeur historique de la capitale changera au début des années 2000.

Figure 2. Un passage commerçant sur la place Wenceslas.

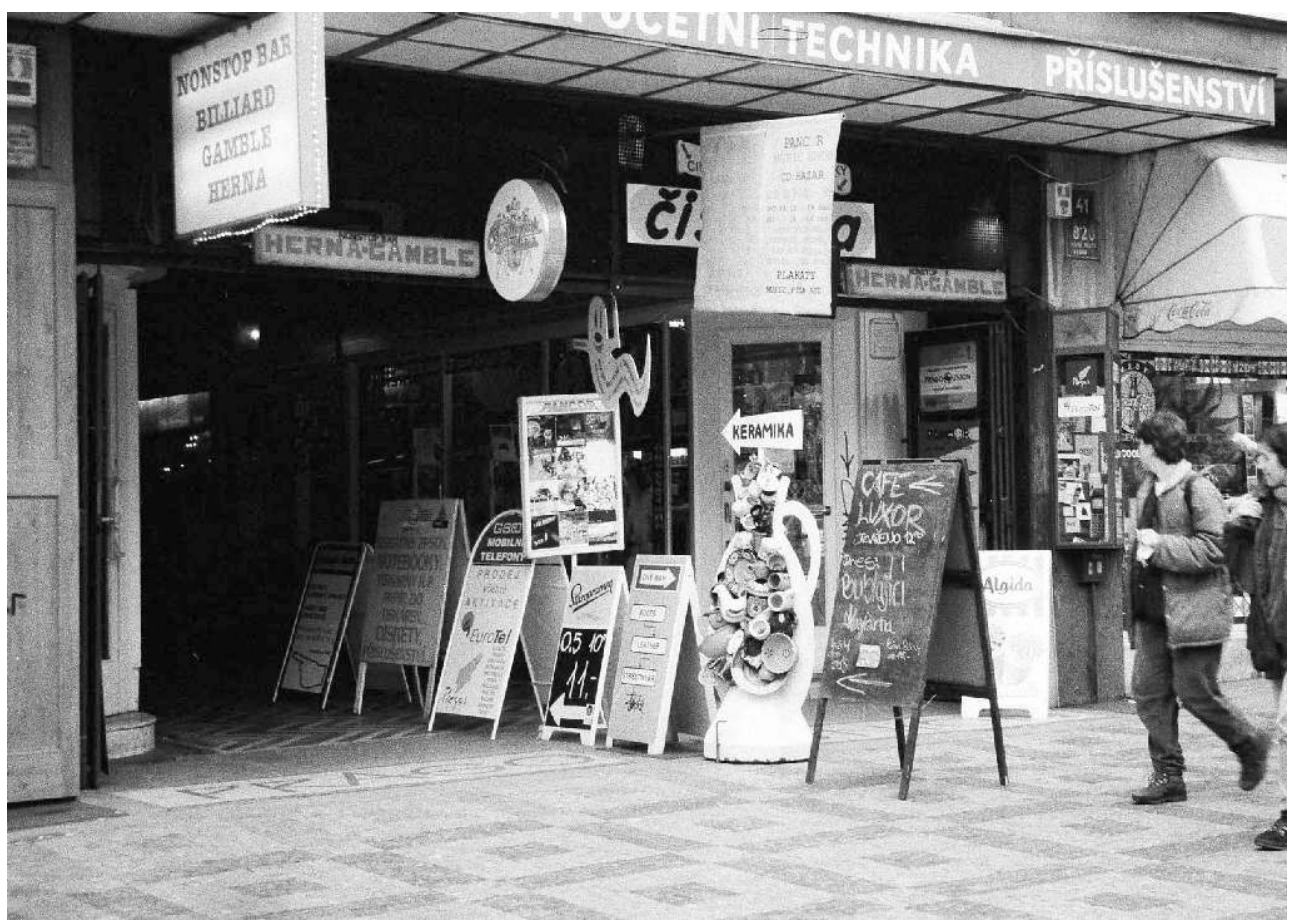

Cliché C. Pommois

11 Le centre-ville de Prague subit ainsi une véritable mutation. Jusqu'au milieu des années 1990, nombre d'immeubles étaient encore obsolètes et menaçaient de s'écrouler. Ceux qui ont connu Prague à cette période se souviennent des échafaudages en bois qui prévenaient les chutes de pierres et qui avaient fini par se fondre dans le paysage urbain : ils ont aujourd'hui disparu. Le dernier d'entre eux, sur la rue Karlova (la voie Charles) qui relie la place de la Vieille Ville au Pont Charles, a survécu jusqu'en 2003. La Vieille Ville se confirme dans sa spécialisation touristique par le nombre des boutiques de souvenirs, des bijouteries, des boutiques de cristal et d'alimentation à emporter. Au Nord de la place de la Vieille Ville, dans le quartier juif, la rue Parizska (avenue de Paris) concentre les boutiques de luxe dont les grands noms de la cosmétique française. Na Prikope et la place Wenceslas se spécialisent dans le prêt-à-porter et continue d'accueillir les enseignes occidentales (Adidas, United Colors of Benetton...). Les petites rues de la Vieille Ville qui, au début de la transition, avaient subi un phénomène de dépréciation et où les immeubles étaient sous occupés, ont changé de visage. Les immeubles sont restaurés, les activités de bureau se sont multipliées, et une spécialisation commerciale se fait jour notamment dans les galeries d'art, les restaurants et les cafés tendance. Tandis que dans la Nouvelle Ville, les activités commerciales restent plus diversifiées et s'adressent à une clientèle essentiellement pragoise (Figure 1).

\section{Des loyers dérégulés}

12 Les valeurs locatives ont aussi évolué vers une différenciation spatiale horizontale et verticale. Les loyers les plus élevés dans le secteur commercial, se trouvent sur $\mathrm{Na}$ 
Prikope, où ils sont comparés à ceux pratiqués dans les capitales occidentales. Ces valeurs décroissent avec l'éloignement vis-à-vis des artères principales. Dans le secteur résidentiel, l'analyse des valeurs locatives est plus délicate du fait de la diversité des situations au sein d'un même immeuble. Au début de la période de transition, les locataires ont bénéficié d'une régulation des valeurs locatives. A partir de 1994, ces résidents ont pu jouir de la possibilité de devenir propriétaire, ce qui, jusque là, était resté un droit restreint par l'obligation de former une association de propriétaires. Dans le même temps, les nouveaux résidents sont soumis au secteur libéral et payent des loyers plus élevés. Cette régulation/dérégulation des valeurs locatives aboutit à des situations diverses au sein d'un même immeuble. Cette variété verticale des loyers rend plus délicate une analyse horizontale. Le lien entre la fonction commerciale et les valeurs locatives du secteur résidentiel n'est pas aisé à étudier.

\section{Des centres commerciaux modernes dans la périphérie pragoise}

13 Jusqu'au milieu des années 1990, les principaux projets de construction (ou projets de développement) consistaient essentiellement en des centres de bureaux, localisés soit sur les marges du centre-ville, soit dans des quartiers périphériques de Prague (Figure 1). A partir de 1994, et plus précisément 1996, les principaux projets concernent le coeur historique (en particulier la Nouvelle Ville) et associent la fonction commerciale avec la fonction des affaires et la fonction administrative. C'est à partir de 1997 que Prague entre dans une nouvelle phase de l'évolution de son organisation spatiale avec l'ouverture du premier centre commercial périphérique, ancré par un hypermarché (de la chaîne suisse Globus) et comprenant une galerie marchande. A partir de cette date, les ouvertures vont se poursuivre. Prague voit naître des centres périphériques de dimension régionale (Figure 3), amorçant la transformation de la capitale de ville mono concentrique en ville polynucléaire. 
Figure 3. Les centres commerciaux à Prague en 2005.

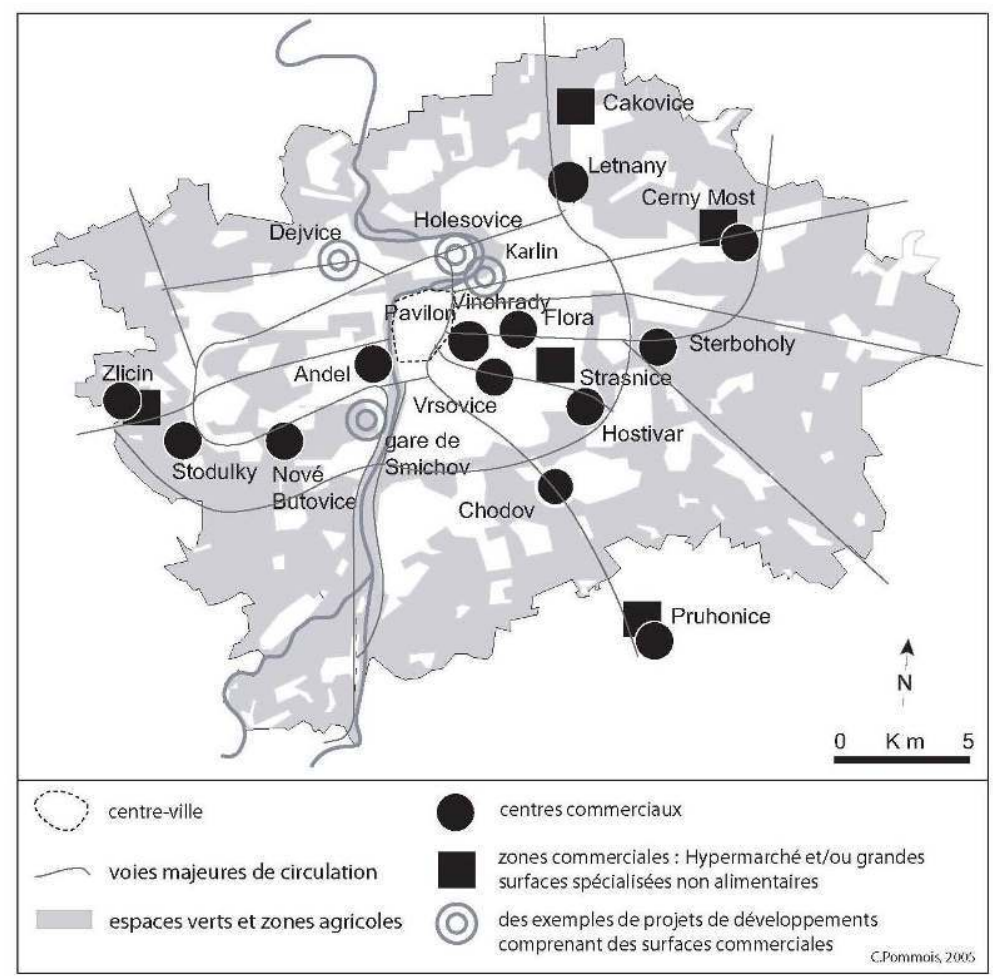
une galerie marchande à passage couvert), soit en parcs commerciaux, rassemblant diverses grandes surfaces spécialisées et des hypermarchés. Parfois, les deux concepts existent côte à côte, mais sans l'apparence d'un agencement planifié, comme à Zlicin à l'ouest de Prague. Ikea, Tesco et d'autres distributeurs forment un ensemble commercial avec passage extérieur, s'organisant autour d'un parking commun. Sur le même site, Globus a ouvert un hypermarché. Plus récemment, en 2003, un centre commercial spécialisé dans le prêt-à-porter, équipé d'un cinéma multiplexe et d'un hypermarché de la chaîne Interspar a ouvert ses portes. rayons multiples dans le centre-ville) ${ }^{5}$, Interspar, Globus et Hypernova ${ }^{6}$ (du groupe Ahold), 3 chacun. Avec les 3 hypermarchés du distributeur français Carrefour, ouverts depuis 2001, Prague totalise 15 hypermarchés. Et si la République Tchèque se caractérise par l'absence de législation restrictive quant au développement des très grandes surfaces, la limitation naturelle à la multiplication des très grands sites commerciaux est l'espace disponible qui vient à manquer dans les périphéries des villes. A Prague, le réseau de supermarchés alimentaires continue son développement.

Les centres de la périphérie constituent une concurrence nouvelle pour le centre-ville historique qui, contrairement aux années 1990, ne constitue plus le premier lieu d'implantation pour les distributeurs occidentaux, notamment dans le secteur du prêt-àporter. Cependant, ces marques qui ouvrent des établissements dans les centres modernes ouvrent en général d'autres magasins dans le centre-ville. Dans les années 2000 , on assiste à une translation du développement commercial de la périphérie vers les faubourgs qui concentrent actuellement les principaux projets de développement. En 
2005, la construction d'un centre commercial, annoncé comme étant «le plus grand » de Prague (ouverture prévue en 2007), viendra renforcer la centralité du centre historique.

Avec l'apparition des très grandes surfaces, forme commerciale inconnue jusqu'en 1997, la concentration de la distribution alimentaire s'accentue. Elle est plus tardive dans le secteur non alimentaire. En même temps que le réseau de supermarchés alimentaires continue de s'accroitre, des grandes surfaces, spécialisées dans le jardinage et l'outillage (Obi, Bauhaus), sont apparues dès la première moitié des années 1990. En 2004, Le vendeur de meubles suédois Ikea a ouvert son deuxième établissement à l'Est de Prague. La concentration de la distribution commence à concerner les petits commerces, surtout à partir du début des années 2000. Dans le secteur du prêt-à-porter, la concentration de la petite distribution se fait au profit des grandes marques ouest-européennes. Dans le secteur alimentaire, on voit la multiplication des initiatives tchèques, notamment dans l'alimentation à emporter.

18 En 2001, un centre commercial, comprenant un hypermarché Carrefour, une galerie marchande essentiellement axée sur l'équipement de la personne, un food court et un cinéma multiplexe, voit le jour sur la marge du centre-ville, dans l'un des premiers quartiers industriels de la fin du XIX ${ }^{e}$ siècle, Smichov. En 2003, un autre centre commercial, cette fois non ancré par un hypermarché, également équipé d'un cinéma multiplexe et d'un food court (Figure 4) ouvre dans la banlieue est de Prague («Flora » sur la Figure 1). Ces deux centres commerciaux suivis, dans la même année, d'un troisième basé sur le même concept, à Zlicin, à l'ouest de Prague, inaugurent l'introduction d'un autre concept, celui du fun shopping. L'activité d'achat est associée à celle de la récréation et des loisirs. Les autres centres commerciaux sont adaptés pour répondre à ce nouvel objectif, lors d'une seconde ou d'une troisième étape du développement du site (les projets comprenant dès le départ plusieurs phases de construction). Dorénavant, les consommateurs ne se rendraient plus dans ces centres uniquement pour y faire des achats, mais aussi pour y rencontrer des amis et se détendre. 
Figure 4. Le premier centre commercial spécialisé dans la mode et la récréation et non ancré par un hypermarché.

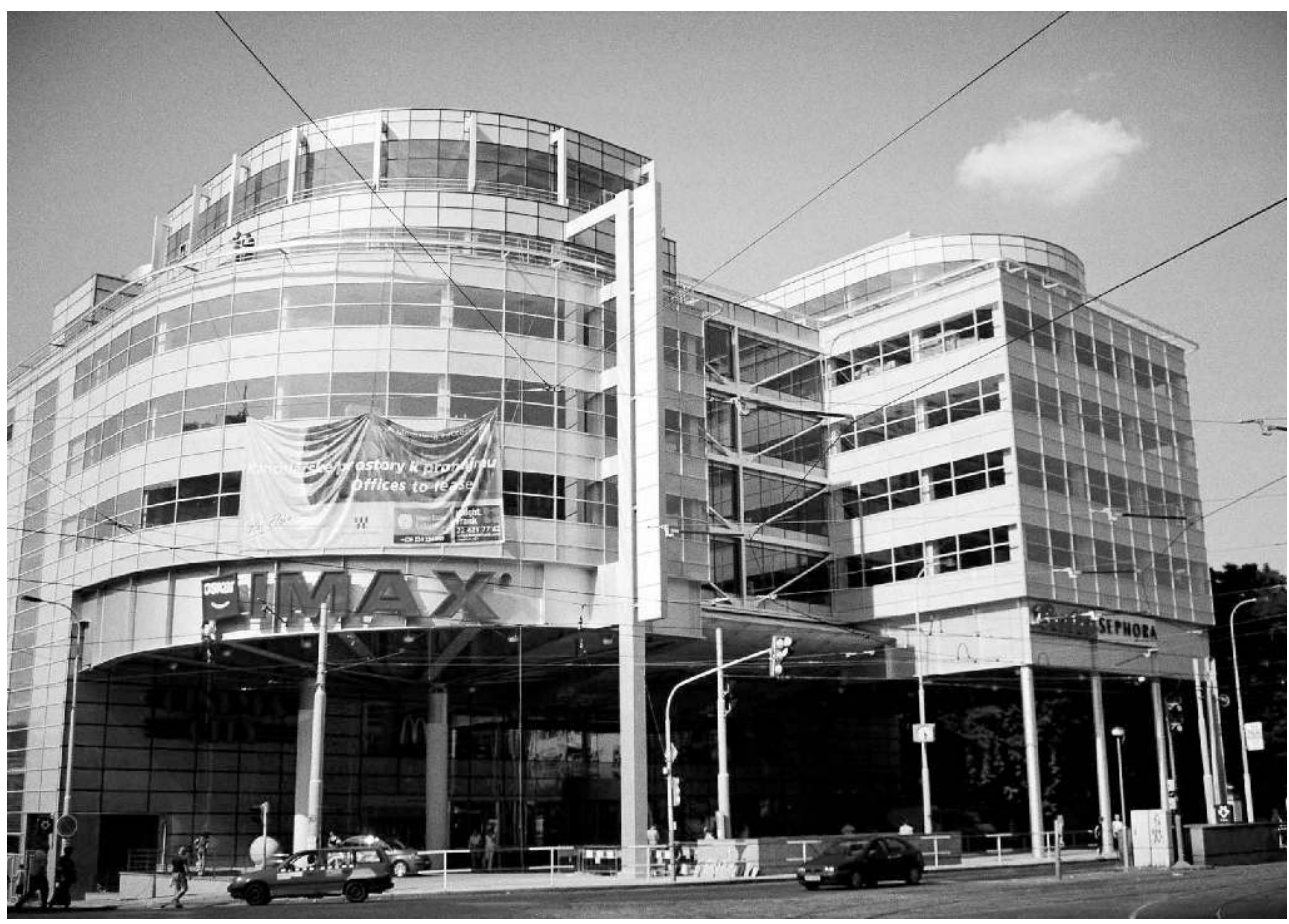

Cliché C. Pommois

Un mouvements urbains convergeaient vers le centre-ville et où ces centres commerciaux constituent des centres périphériques concurrençant le centre-ville traditionnel. Les centres commerciaux de la périphérie sont de nouveaux pôles d'attraction, notamment le week-end et les jours fériés. Beaucoup de Pragois quittent la capitale pour le repos dominical, cependant, la fréquentation des commerces ouverts dans les artères majeures $\mathrm{du}$ centre historique et des centres commerciaux modernes est importante. Dans le centre-ville, les commerces, localisés hors des artères principales et hors de la zone touristique, sont, pour la plupart, fermés le week-end. Le centre historique reste surtout le domaine des touristes les samedis, dimanches et jours fériés, tandis que les centres commerciaux sont très largement fréquentés par les Tchèques. Dans les autres quartiers de la ville, seuls quelques commerces de proximité sont ouverts le dimanche. Les centres modernes participent à l'évolution des pratiques urbaines et certainement, comme l'on a pu le constater pour une large partie de la population, à une modification du temps de 
loisirs et de récréation. Les centres commerciaux constituent de nouveaux lieux de socialisation.

\section{Les nouveaux comportements d'achat des Pragois}

\section{Le rêve tchèque}

31 mai 2003, 10 heures, un ciel orageux. De nombreux Pragois se rendent à la périphérie, au nord de Prague afin d'assister à l'ouverture d'un tout nouvel hypermarché Cesky Sen, le Rêve Tchèque en français. A Cesky Sen, on ne trouve que des produits tchèques à très bas prix. L'inauguration commence, le ruban est coupé, les premiers clients se pressent vers l'hypermarché, là-bas, deux cents mètres plus loin. Il n'y a pas de parking, et c'est un sentier qui mène à l'entrée du magasin, planté au milieu d'un immense champ herbé (en fait la piste d'un aérodrome). Les premiers, les plus rapides, arrivent. Ils cherchent l'entrée, contournent le bâtiment et... Stupéfaction, ce n'est qu'un décor de théâtre. La façade colorée arc-en-ciel de Cesky Sen, n'est qu'une immense toile soutenue par un échafaudage (figure5). Déception, les premiers arrivés s'en retournent tandis que les suivants continueront d'affluer pour finalement découvrir la supercherie. Ceux qui reviennent préviennent ceux qui arrivent, et qui, malgré cela, iront jusqu'au bout pour voir de leurs propres yeux. Cesky Sen n'était qu'une immense campagne publicitaire, produite par deux étudiants en cinématographie. Depuis quelques semaines, des affiches publicitaires rouge et bleue étaient placardées dans les stations de métro. Un site Internet vantait les bas prix des articles entièrement de production tchèque. L'on pouvait même écouter un hymne, composé spécialement pour cet hypermarché qui finalement n'est qu'un rêve. Car il s'agit bien de cela. Cesky Sen se présente comme une dénonciation de la société de consommation et de la globalisation. La qualité tchèque, en plus de la faiblesse des prix, a réussi à attirer de nombreux Pragois. Pourtant, les affiches publicitaires indiquaient bien qu'il ne fallait pas y aller. Le lieu était même resté secret jusqu'à quelques semaines avant son ouverture. Mais à force de lire « ne faites pas ci », « n'y allez pas ", nombreux sont ceux qui ont mordu à l'hameçon: des personnes âgées, certaines pouvant à peine se déplacer; des familles entières qui se sont déplacées en voiture, d'apparence plutôt modeste. Les autorités de la ville s'étaient rendues complices de ce canular. Toute la journée durant, des bus des transports urbains pragois ont assuré la navette entre la station de métro la plus proche (quelques kilomètres tout de même) et Cesky Sen. Et toute la journée durant, les gens se sont déplacés. Surprenante a été leur réaction lorsque Cesky Sen s'est révélé n'être vraiment qu'un rêve, le rêve de personnes, exclues de la consommation moderne, attirées par les faibles prix. On sourit, on se justifie de s'être laissé berner, on se dit "je le savais mais il fallait que je le voie", ou "c'est ça l'Europe »... quand ce n'est pas le Président de la République Tchèque qui est accusé. Les gens sont restés finalement assez philosophes bien qu'il y ait eu des déceptions énormes ${ }^{7}$. En 2004, le film des deux étudiants est sorti dans les salles. Les images sont assez choquantes. L'on voit ces dames âgées se déplaçant à grand peine à l'aide de béquilles, des familles déçues parce qu'elles n'ont pu trouver le téléviseur à 500 couronnes (l'équivalent de 15 euros) promis dans le prospectus. Le film montre aussi une famille, poussant un caddie plein et chantant un canon à tue-tête, scène stigmatisant la société de consommation. Il a été intéressant de noter que le public qui s'est rendu dans les salles de cinéma pour voir ce film, n'est pas le même que celui qui était sur les lieux un an 
auparavant ${ }^{8}$. Cette expérience met à jour l'existence d'une population modeste, sensible à la publicité et aux bas prix, exclue de la consommation moderne.

Figure 5. L'hypermarché Cesky Sen : un décor pour un projet cinématographique en 2003.

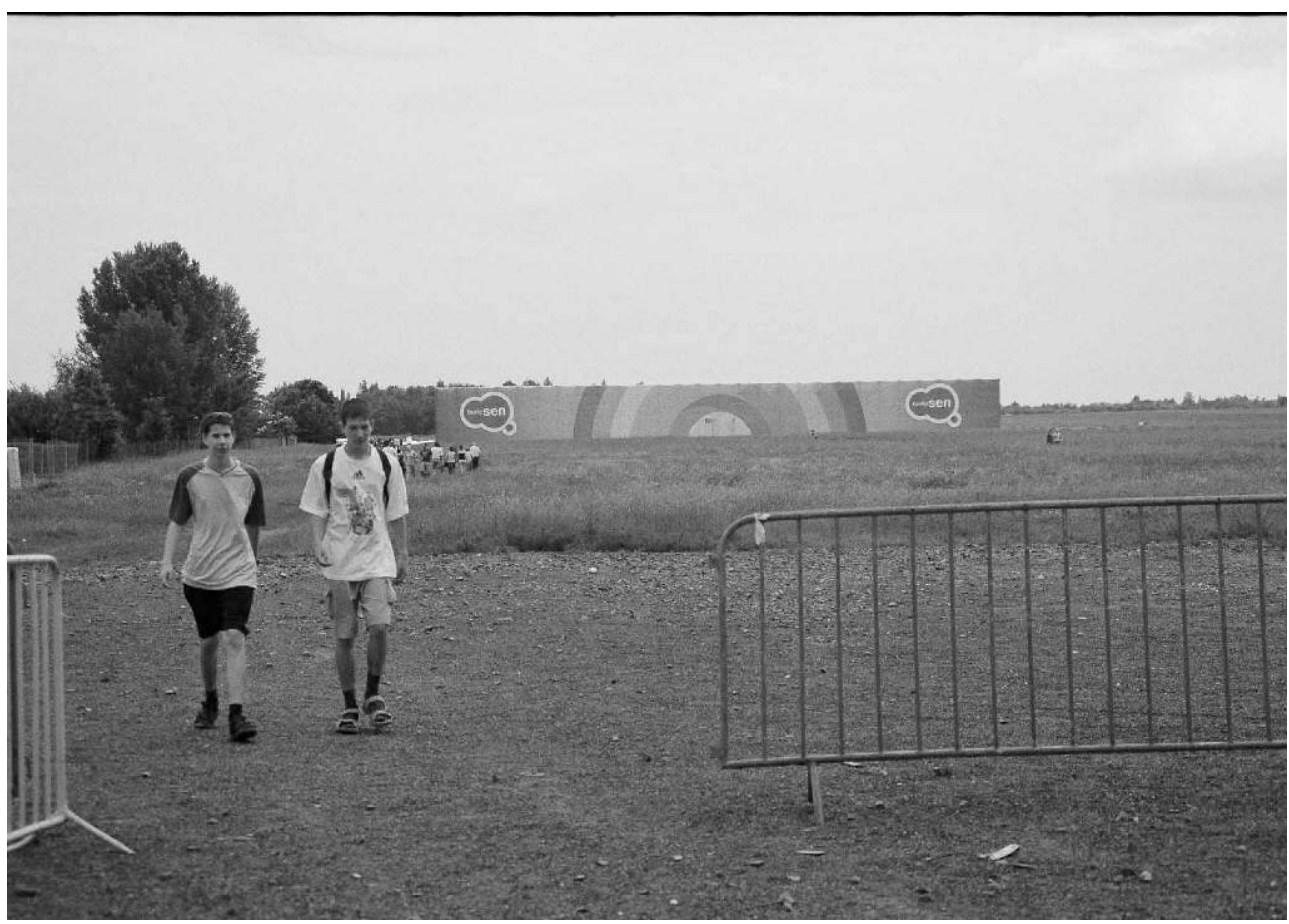

Cliché C.Pommois

\section{Les habitudes d'achat entre héritage et emprunts}

Les Tchèques, à la sortie du régime communiste, ont découvert la société de consommation. Mais c'est avec l'apparition des hypermarchés et des centres commerciaux, shopping malls en anglais, que les habitudes d'achat vont évoluer. Avec l'arrivée de ces nouvelles formes commerciales et des concepts qu'elles introduisent, tels que le fun shopping et l'out shopping, l'on observe une occidentalisation des comportements d'achat, une volonté de la part des Tchèques d'adopter un mode de vie occidental. Les principales études menées par des sociétés de marketing ont montré une augmentation annuelle de la fréquentation des hypermarchés dans l'ensemble de la République Tchèque. La problématique de la recherche exposée ici est celle de l'identification de divers types de comportement spatial des consommateurs pragois, notamment à propos des hypermarchés et des centres commerciaux qui représentent l'introduction physique de la consommation de masse et du fun shopping, sur la base d'une enquête, réalisée au cours de l'été 2004, sur un échantillon de 400 personnes.

Ces personnes ont été questionnées sur leur comportement d'achat dans le domaine des achats de biens de consommation courante. Nous leur avions demandé de citer les commerces qu'elles avaient fréquentés au cours du dernier mois et de préciser le type de commerce dont il s'agissait. Il apparaît que la forme commerciale la plus citée est le supermarché (cité 412 fois); les hypermarchés ont été cités 343 fois, très loin devant les petits commerces traditionnels (117) (Tableau 1). Au cours de ce même dernier mois précédant l'enquête, $67 \%$ des répondants ont fait des achats alimentaires dans des 
supermarchés, et $58 \%$ ont visité des hypermarchés tandis que seuls $25 \%$ ont visité des petits commerces traditionnels. Ces résultats viennent confirmer ceux obtenus par les sociétés de recherche en marketing, mettant en avant la préférence des Pragois pour les supermarchés plutôt que les hypermarchés dans le secteur des achats alimentaires. Cette tendance vient souligner la préférence stratégique des distributeurs pour les petites surfaces tandis que, de la fin des années 1990 jusqu'au début des années 2000, le développement des hypermarchés était à son apogée. Et si les Pragois fréquentent de plus en plus les hypermarchés, ils n'y font pas forcément des achats importants. Une observation des caddies à la caisse l'atteste. Les Pragois dépensent peu d'argent dans les hypermarchés.

Tableau 1. Les formes commerciales fréquentées pour les achats courants (dont l'alimentation).

\begin{tabular}{|l|c|}
\hline \multicolumn{1}{|c|}{ Forme commerciale } & Nombre de citations \\
\hline Le supermarché & 412 \\
\hline L'hypermarché & 343 \\
\hline Le petit commerce & 117 \\
\hline La supérette & 94 \\
\hline Le marché & 27 \\
\hline Le centre commercial & 11 \\
\hline $\begin{array}{l}\text { La grande surface spécialisée dans la maison - outillage - } \\
\text { jardinage }\end{array}$ & 5 \\
\hline Le grand magasin spécialisé & 3 \\
\hline Le grand magasin à rayons multiples & 2 \\
\hline
\end{tabular}

Les personnes ont également été interrogées sur la fréquence de leurs achats. $26.5 \%$ d'entre elles affirment faire des achats deux à trois fois par semaine, $24.5 \%$ quotidiennement, $21.3 \%$ une fois par semaine, et $20.5 \%$ quatre à six fois par semaine. Il apparaît nettement que la majorité des répondants ont conservé un comportement traditionnel. Seuls $6.2 \%$ font des achats une fois tous les 15 jours et $1 \%$ déclarent faire des achats une fois par mois. Ces répondants ont pu être classés en tenant compte de leur fréquentation des hypermarchés (le supermarché ayant dès le début des années 1990 remplacé l'épicerie traditionnelle) et la fréquence de leurs achats (Tableau 2). Il apparaît que plus de la moitié de notre échantillon ( $57 \%$ des répondants) fréquente les hypermarchés et néanmoins fait ses achats plusieurs fois par semaine. 17 \% fréquentent les hypermarchés et réalisent leurs achats une fois par semaine. Plus rares sont ceux qui font leurs achats moins d'une fois par semaine tout en fréquentant les hypermarchés (5.7\%). Ce résultat vient appuyer une observation qui a pu être faite dès la première apparition des hypermarchés, à savoir que nombre de Pragois ont réalisé un transfert de leur comportement traditionnel de la petite surface ou du petit commerce vers la très grande surface. Force est de constater qu'encore de nos jours, le panier reste très limité. On se rend dans un hypermarché pour faire des petits achats, parfois même ses achats alimentaires pour la journée. Ce transfert des habitudes d'achat est facilité par le fait qu'à Prague, tous les hypermarchés et centres commerciaux de la périphérie sont accessibles par les transports en commun. Quand ces centres ne sont pas desservis par le métro, des navettes assurent la liaison régulière entre la station la plus proche et le centre commercial. Le report des habitudes d'achat vers les très grandes surfaces est encore facilité par la présence dans le tissu intra urbain d'un centre commercial ancré par un hypermarché, bâti sur deux niveaux. Ce centre se trouve à l'un des axes majeurs de 
Prague, dans l'un des faubourgs industriels de la fin du XIX ${ }^{\mathrm{e}}$ siècle et qui, selon les habitudes pragoises, est considéré comme appartenant au centre de Prague.

Tableau 2. Une typologie des consommateurs pragois selon la visite des hypermarchés et la fréquence des achats.

\begin{tabular}{|l|l|c|c|}
\hline \multicolumn{2}{|l|}{ Groupes } & \multicolumn{1}{c|}{$\begin{array}{c}\text { Nombre de } \\
\text { répondants }\end{array}$} & $\begin{array}{c}\% \text { de } \\
\text { répondants }\end{array}$ \\
\hline \multirow{3}{*}{ Fréquente les hypermarchés } & Plusieurs fois par semaine & 228 & 57.00 \\
\cline { 2 - 4 } & Une fois par semaine & 68 & 17.00 \\
\cline { 2 - 4 } & $\begin{array}{l}\text { Moins d'une fois par } \\
\text { semaine }\end{array}$ & 23 & 5.75 \\
\hline $\begin{array}{l}\text { Ne fréquente pas les } \\
\text { hypermarchés }\end{array}$ & Plusieurs fois par semaine & 58 & 14.50 \\
\cline { 2 - 4 } & Une fois par semaine & 17 & 4.25 \\
\cline { 2 - 4 } & $\begin{array}{l}\text { Moins d'une fois par } \\
\text { semaine }\end{array}$ & 6 & 1.50 \\
\hline Nombre total de répondants & & 400 & 100.00 \\
\hline
\end{tabular}

L'apparition des centres commerciaux et des hypermarchés a également modifié les comportements spatiaux des consommateurs. Les quartiers ayant vu l'implantation de ces formes commerciales modernes ont également connu un accroissement de leur fréquentation. Toujours dans le domaine des achats alimentaires, le quartier qui a été le plus cité est Andel localisé dans Smichov (cité 113 fois). Le centre-ville historique est cité 111 fois. A l'intérieur même du centre-ville, c'est le quartier Nové Mesto (Ville Nouvelle) qui a été nommé 100 fois contre 11 fois pour le quartier de la Vieille Ville qui, comme nous l'avons souligné, est spécialisé dans la fonction touristique. Les centres commerciaux de la périphérie sont largement cités: de 31 fois pour Stodulky (sa proximité avec le centre commercial de Zlicin qui comprend trois hypermarchés, une galerie marchande, un cinéma multiplexe, des grands magasins spécialisés, peut expliquer que le centre de Stodulky ait une aire de recrutement plus locale) à 70 fois pour Zlicin. $34.3 \%$ des personnes interrogées estiment avoir changé leurs habitudes d'achat depuis l'ouverture des centres commerciaux et des hypermarchés.Les proportions sont semblables de ceux visitant moins les commerces de leur rue (31,5\%), de leur quartier $(31,7 \%)$ ou du centre-ville $(36,8 \%)$. Très peu déclarent y avoir augmenté leurs visites (6\% pour les commerces de leur quartier et $2 \%$ pour ceux de leur rue et du centre-ville); les autres n'ont rien changé. En ce qui concerne les achats alimentaires, la distance maximale parcourue (à vol d'oiseau) est approximativement de $18 \mathrm{Km}$. La distance moyenne parcourue pour l'ensemble de l'échantillon est de $5 \mathrm{Km}$. 49 répondants sur les 400 fréquentent exclusivement les commerces de leur quartier. Avec le centre commercial, le concept d'out shopping apparaitt à Prague. Le week-end, on peut observer l'affluence dans les principaux centres commerciaux périphériques.

\section{Des nouvelles pratiques urbaines}

Lors d'interviews informelles avec des Pragois, une réflexion courante a été celle-ci : « Maintenant, les gens vont dans les centres commerciaux les samedis après-midi, au lieu de se promener dans les parcs, comme avant ». En effet, la question de savoir si les centres commerciaux modernes deviennent des lieux de socialisation se pose aussi bien en République Tchèque qu'en France. L'enquête s'intéresse aussi au comportement des habitants de la capitale vis-à-vis des centres commerciaux modernes, à savoir quelles sont leurs activités lorsqu'ils visitent un centre commercial. Au vu des résultats de l'enquête, il apparaît que $18 \%$ des Pragois ne fréquentent jamais les centres commerciaux. $24 \%$ les 
fréquentent moins d'une fois par mois, $19.5 \%$ une fois par mois, $17 \%$ une fois toutes les deux semaines et $12.5 \%$ une fois par semaine. Le reste de l'échantillon, soit $9 \%$ des répondants, les visitent plusieurs fois par semaine.

La majorité des répondants ( $55 \%$ ) déclarent se rendre dans un centre commercial en compagnie de leur partenaire. Dans une proportion plus faible, les répondants sont accompagnés de leurs enfants (39\%) ou de leurs amis (42.5\%). La raison première de la visite des centres commerciaux est l'hypermarché puisque $79.75 \%$ des répondants y font leurs courses. Plus de la moitié de notre échantillon y fréquente les restaurants et la proportion de ceux qui s'y arrêtent pour un café est légèrement supérieure à celle des répondants qui déclarent ne jamais y prendre un café (42.5\% contre $38.75 \%$ ). Il en est de même pour la fréquentation du cinéma (Tableau 3). Près de la moitié des répondants fréquente aussi les centres commerciaux pour y exercer d'autres activités relevant de la socialisation et de la récréation, soit en famille soit avec des amis. Ces résultats permettent d'avancer l'hypothèse que le centre commercial moderne est en train de devenir un lieu de récréation au même titre que les centres urbains traditionnels. Plus de la moitié des répondants $(59.75 \%)$ fréquente les centres commerciaux le samedi et $52.5 \%$ les visitent le dimanche. $64.75 \%$ des répondants en profitent également pour faire les boutiques. Cela signifierait que le week-end, près de la moitié de la population pragoise se rend dans ces centres commerciaux, d'où la critique, souvent formulée, que ces formes commerciales modernes s'accompagnent d'une modification même des loisirs, alors que traditionnellement, les Pragois qui passent leur week-end dans la capitale, avaient pour coutume de se promener dans les parcs qui entourent la ville. Les centres commerciaux sont souvent critiqués par les Pragois (il y a trop de monde, c'est trop cher), pourtant la grande majorité d'entre eux les fréquentent malgré tout. Les centres commerciaux exercent une attraction; ils représentent l'expression du mode de vie occidental qui se résume par la société de consommation, tout en provoquant un phénomène de répulsion. Lors de mes interviews informelles, il apparaissait clairement que ceux qui critiquaient ces formes commerciales les fréquentaient aussi régulièrement. Par contre, les autres formes de commerces (correspondance, catalogue, Internet, Télé-achat) ont encore du mal à faire leur percée dans les nouvelles habitudes d'achat. Seule une très faible minorité des personnes interrogées lors de cette enquête a déclaré avoir eu recours à ces formes commerciales. La raison invoquée est celle, entre autre, de la méfiance vis-à-vis des services de la poste. Le colis n'est pas certain de parvenir à destination'. 
Tableau 3. La fréquentation des centres commerciaux ( \%).

\begin{tabular}{|l|l|l|c|c|}
\hline Quand je visite un centre commercial, il m'arrive : & non & oui & sans réponse & total \\
\hline D'y aller seul(e) & 25.50 & 58.75 & 15.75 & 100 \\
\hline D'y aller avec mon (ma) partenaire & 28.00 & 55.00 & 17.00 & 100 \\
\hline D'y aller avec mes enfants & 44.00 & 39.00 & 17.00 & 100 \\
\hline D'y rencontrer des amis & 41.50 & 42.50 & 16.00 & 100 \\
\hline D'y faire mes courses & 3.00 & 79.75 & 17.25 & 100 \\
\hline D'y manger & 26.25 & 55.75 & 18.00 & 100 \\
\hline De prendre un café & 38.75 & 42.50 & 18.75 & 100 \\
\hline D'en profiter pour faire les boutiques & 19.00 & 64.75 & 16.25 & 100 \\
\hline D'aller au cinéma & 40.25 & 42.75 & 17.00 & 100 \\
\hline D'aller chez le coiffeur & 74.25 & 7.75 & 18.00 & 100 \\
\hline D'aller au centre sportif & 67.25 & 15.00 & 17.75 & 100 \\
\hline D'y aller le samedi & 23.00 & 59.75 & 17.25 & 100 \\
\hline D'y aller le dimanche & 30.25 & 52.50 & 17.25 & 100 \\
\hline D'y aller en semaine & 13.00 & 69.50 & 17.50 & 100 \\
\hline
\end{tabular}

28 Avec les hypermarchés et les centres commerciaux, un autre phénomène a fait son apparition : la consommation à crédit, largement encouragée depuis quelques années par les banques maintenant privatisées et à capital occidental, et par les distributeurs euxmêmes, qui développent la formule de la carte de fidélité. A côté de ces deux acteurs, il en est apparu un troisième : la société privée de prêt qui propose des crédits mais à des taux élevés. Il était possible jusqu'en 2004 de cumuler différentes formes de prêts et d'en souscrire dans plusieurs banques. Cependant, face à l'ampleur croissante de ce phénomène et des clients non solvables, un système de contrôle avec partage des banques de données a été mis en place, permettant aux banques de vérifier la solvabilité de l'emprunteur, et si celui-ci ne s'est pas déjà endetté dans un autre établissement. Les banques ont aussi développé des prêts spécifiques pour les étudiants. Une carte de paiement a été élaborée spécifiquement pour eux, leur permettant de bénéficier de réductions dans des établissements commerciaux et cinémas qui ont signé un partenariat avec la banque en question ${ }^{10}$. On peut tout acheter à crédit et les Tchèques y recourent de plus en plus. On aboutit à des situations de surendettement. Si le phénomène est encore loin d'atteindre les niveaux connus en France par exemple, ce qui inquiète les économistes tchèques est sa rapidité. Il n'y a pas trois ans encore, on pouvait lire dans la presse spécialisée que les Tchèques n'avaient pas pour habitude de dépenser l'argent qu'ils ne possédaient pas ${ }^{11}$.

\section{Conclusion}

Les hypermarchés et centres commerciaux périphériques représentent une concurrence sérieuse pour les commerces du centre-ville, en particulier pour les grands magasins, symbole du commerce de détail des années 1980, et pour le petit commerce alimentaire. Dans le centre-ville, les grands magasins ont des difficultés à soutenir cette concurrence nouvelle (quand ces difficultés ne sont pas dues à des problèmes de gestion). Quant au petit commerçant indépendant, il doit redoubler d'imagination pour se maintenir. Sa clientèle est vieillissante et se raréfie. Il doit privilégier le contact personnel avec le client. D'ailleurs, les épiceries traditionnelles ont disparu du paysage commercial de la ville. Le petit commerce alimentaire qui existe est un commerce spécialisé. Les Pragois ne fréquentent plus guère les supérettes. Celles du centre-ville se spécialisent dans 
l'alimentation de luxe et l'alimentation à emporter. Sur les marges du quartier touristique, il subsiste quelques commerces alimentaires spécialisés dont la clientèle est principalement celle du centre-ville. Dans les autres quartiers de la ville, on observe le même phénomène. Quant aux marchés alimentaires, ils se réduisent de plus en plus, ces marchés étant en très grande majorité tenus par les vietnamiens.

Enfin, il faut noter l'ouverture courant 2004 de plusieurs établissements d'une même chaîne de discount. Nombreux sont les Pragois à les avoir visité dès leur ouverture. Le développement du discount, s'il se poursuit, pourra poser des difficultés aux chaînes de supermarchés et hypermarchés, d'autant plus que les acheteurs tchèques sont très attentifs aux prix (ayant même tendance à les tirer vers le bas, comme l'ont pu le constater des grands distributeurs à Prague). Le paysage commercial continue de se dessiner. Julius Meinl, chaîne de supermarchés alimentaires " haut de gamme ", a disparu au profit de la chaîne Albert qui poursuit son expansion. Le paysage des enseignes commence à se simplifier.

31 Alors que depuis la fin des années 1990, les projets concernaient essentiellement la périphérie de la ville, le centre-ville historique voit sa centralité se renforcer avec la construction du «plus grand centre commercial de la ville», dont l'ouverture est prévue pour 2007. D'autres projets incluant les fonctions commerciales, résidentielles et d'affaires, seront réalisés dans les faubourgs de Prague. Au vu des nombreux projets urbains en cours ou en attente des autorisations nécessaires, il faudra bien encore une décennie pour que l'organisation spatiale de Prague commence à se stabiliser.

\section{BIBLIOGRAPHIE}

BLAHA J. (1987), « Tchécoslovaquie 1986-1990 : à petits pas », Le Courrier des Pays de l'Est, 316, mars 1987, pp. 54-59.

BLAHA J. (1989), « Vers un marché noir unique à l'Est, l'essor du tourisme de shopping », Le Courrier des Pays de l'Est, 342, août-septembre 1989, pp. 40-48.

BLAHA J. (1990), «L'économie tchécoslovaque en 1989-1990 : la marche vers l'Ouest », Le Courrier des Pays de l'Est, 349, avril 1990, pp. 75-83.

BURGEL G. (1993), La ville aujourd'hui, Hachette, Pluriel, 224 p.

CARTER F.W. (1979), « Prague and Sofia : an analysis of their changing internal city structure », in FRENCH R.A \& HAMILTON F.E.I., The socialist city ; spatial structure and urban policy, edition French \& Hamilton, pp. 425-460.

CFCE (1997), Un marché, République Tchèque, les éditions du CFCE, janvier 1997, 198 p.

CHALINE C. (1980), La dynamique urbaine, A. Colin, coll. U2, 254 p.

DRTINA T. (1997), The internationalization of retailing in the Czech and Slovak Republics, Frank Cass Journals, London, pp. 191-203. 
EARLE J.S. et al. (1994), Small privatization; the transformation of retail trade and consumer services in the Czech Republic, Hungary and Poland, Central European University Press, 301 p.

FOUCHER M. (1993), « Fragments d'Europe », The Prague Business Journal, Haeley \& Baker, Prague, Bureau de Développement de la Ville de Prague, 12-18 janvier 1998.

FRENCH R.A. \& HAMILTON E.I. (1979), « Is there a socialist city? », in FRENCH R.A \& HAMILTON F.E.I., The socialist city; spatial structure and urban policy, edition French \& Hamilton, pp. 1-22.

GABRISCH H. (1989), « Réformes et politiques des prix à l'Est », Le Courrier des Pays de l'Est, 342, août-septembre 1989, pp. 49-58.

KAPLAN J., NOSARZEWSKA K. (1997), Prague, Könemann Verlagsgesellschaft mbH, 360 p.

PAAL M. (1996), « La transformation à Prague et Budapest : la mutation commerciale », in Nouveaux espaces et systèmes urbains, Mobilité Spatiale, SEDES, pp. 199-216.

PLICKA I. (1997), Prague 97 metropolitan area report urban development of the city, City Development Authority, Prague, $75 \mathrm{p}$.

POMMOIS C (2004), « La consommation à Prague, Impacts sur l'espace urbain », Etudes du CEFRES $N^{\circ}$ 2, Prague, septembre 2004, 36 p.

POMMOIS C. (2004), « The retailing urban structure of Prague from 1990 to 2003 : catching up with the western cities? », European Spatial Research and Policy, International Journal, Poland, Lodz, 2004/1, pp. 117-133.

POMMOIS C. (2001), Les villes d'Europe centrale et orientale, du socialisme au libéralisme. Analyse du commerce de détail d'une capitale en mutation, Prague (République Tchèque), Thèse de doctorat de l'Université Louis Pasteur de Strasbourg et de l'Université Charles de Prague, 445 p.

POMMOIS C. (1999), « Dynamique urbaine à Prague de la fin de la période communiste à nos jours : l'importation du modèle occidental », Revue Géographique de l'Est, XXXIX, 2-3, pp. 97-106.

SCHULTZ J. (1997), « Mutations des structures et espaces urbains : les capitales de l'Europe médiane », in MAUREL M.C, Recomposition de l'Europe médiane, SEDES, pp. 10-17.

SYKORA L. (1999), " Changes in the internal spatial structure of post-communist Prague ", GeoJournal 49(1), pp. 79-89.

SYKORA L. (1997), « Prague, mutations économiques et sociales », Le Courrier des Pays de l'Est, 416, janvier-février 1997, pp. 24-39.

\section{NOTES}

1. Source: The Prague Post, 1993.

2. Rebaptisé depuis « Bureau de Développement de la Ville de Prague ».

3. Afin de protéger en quelque sorte les habitants de la ville, les loyers n'ont pas été libéralisés et les locataires qui vivaient dans leur logement en 1989 peuvent ainsi continuer à y vivre en échange d'un loyer dérisoire. Cette mesure protectrice n'est pas un avantage pour un nouveau propriétaire, ce dernier ne récoltant aucun bénéfice et ne disposant ainsi pas de moyens pour entretenir l'immeuble. Ces propriétaires voyant l'arrivée des firmes occidentales comme une source de profit (puisque pour les nouveaux arrivants, on applique les loyers à valeur du marché), vont alors élaborer diverses stratégies pour inciter les résidents actuels à quitter de leur propre chef leur logement. En effet, si le propriétaire rompt le contrat de location, celui-ci doit payer les frais d'un déménagement et trouver pour ses locataires, qu'il voudrait remplacer 
par des bureaux ou des locataires étrangers prêts à payer plus, un logement équivalent. Pour le reste, les contrats de bail issus de la période communiste sont d'une très longue durée (plus de 90 ans) et sont transmissibles par héritage si les héritiers en question résident aussi dans le logement.

4. Le dernier établissement Tesco de Prague a ouvert en novembre 2005.

5. A la fin du mois de septembre 2005, le distributeur anglais Tesco a confirmé qu'il effectuera à la fin de l'année 2005, un échange avec la chaîne française Carrefour. Tesco récupère 11 hypermarchés Carrefour en République Tchèque et 4 en Slovaquie, en échange de 8 hypermarchés et sites Tesco à Taïwan (Source : Radio Prague, septembre 2005). La domination de la chaîne de distribution anglaise dans le paysage commercial de Prague s'en retrouve confortée. Avec le départ de Carrefour, les Français sont absents de la grande distribution en République Tchèque, alors qu'ils sont bien présents dans d'autres pays d'Europe Centrale, notamment en Pologne, Hongrie et Roumanie. Le secteur de la grande distribution tchèque est occupé par les Anglais, les Allemands, les Autrichiens, les Suisses et les Belges.

6. Le dernier établissement Hypernova a ouvert en novembre 2005.

7. J'étais personnellement présente sur les lieux, dans la matinée et dans l'après-midi du 31 mai 2003. Ce paragraphe est basé sur de l'observation directe de terrain.

8. Ce fait est basé sur de l'observation directe dans un cinéma du centre-ville de Prague.

9. Source : The Prague Tribune (2000-2004).

10. Cette opération avait commencé en avril 2003 et, à la fin de la même année, près de 120000 cartes avaient été délivrées. Source : The Prague Tribune.

11. Source: The Prague Tribune (2000-2004).

\section{RÉSUMÉS}

L'organisation spatiale du commerce de détail à Prague évolue très rapidement. Depuis 1997, les centres commerciaux modernes se sont multipliés, et la dernière génération d'entre eux introduit le concept de fun shopping. Pour les professionnels du marketing, il s'agit de rendre l'activité d'achat plus agréable en l'associant à la récréation et la socialisation. Les habitants de Prague modifient leurs comportements d'achat en empruntant le mode de vie à l'occidentale, ou du moins l'image qu'en ont les Pragois, et qui s'identifie par la société de consommation. Au courant de l'année 2004, une enquête a été réalisée auprès de 400 habitants de Prague. Le but de cette étude était l'identification de divers types de comportement spatial par l'analyse des déplacements à but d'achat et de mesurer le rôle des centres commerciaux comme lieu de socialisation. Les premiers résultats sont présentés dans cet article.

The retailing spatial organization of Prague evolves with rapidity. Since the opening of the first modern shopping centre in the outer-suburbs of the Czech capital city, shopping centres and hypermarkets spread, and the last generation introduces, in the city, the concept o fun shopping. For professionals, the shopping activity must be combined with leisure and recreation. Today, modern shopping malls provide food courts, multiplex cinemas, fitness centres. Inhabitants of Prague are changing their shopping habits and are adopting a western way of life that is identified with the consumption society. In 2004, a sample of 400 inhabitants, located within Prague, has been interviewed. The goal of the survey was the definition of different kinds of 
shopping behaviours and the definition of the role of shopping centres as new location for socialization. The first results of this survey are presented in this paper.

INDEX

Mots-clés : Prague, commerce de détail, comportement spatial des consommateurs, fun shopping, communisme, tourisme

Keywords : retail trade, consumers' spatial behaviour, communism, tourism

\section{AUTEUR}

\section{CAROLE POMMOIS}

Peugeot Ceska Republika sro, Na strzi 40, 14000 Prague 4, Czech Republic, carole.pommois@peugeot.com 